\title{
Bose condensation of nuclei in heavy ion collisions
}

\author{
Ram K. Tripathi \\ Christopher Newport University, Newport News, Virginia 23606 \\ Lawrence W. Townsend \\ NASA Langley Research Center, Hampton, Virginia 23681
}

(Received 10 January 1994)

\begin{abstract}
Using a fully self-consistent quantum statistical model, we demonstrate the possibility of Bose condensation of nuclei in heavy ion collisions. The most favorable conditions of high densities and low temperatures are usually associated with astrophysical processes and may be difficult to achieve in heavy ion collisions. Nonetheless, some suggestions for the possible experimental verification of the existence of this phenomenon are made.

PACS number(s): $25.70 .-\mathrm{z}$
\end{abstract}

For some time, there has been an intrinsic interest in Bose condensation of particles with a concomitant phase transition in nuclear physics. Studies of multifragment final states over a broad range of incident energies may provide information about liquid-vapor and other types of nuclear phase transitions, and ultimately information about the equation of state of nuclear matter [ $1-12]$, provided that multifragment disintegrations are related to bulk instabilities of nuclear matter at low densities. Most experimental observations [8] provide end-point information and do not necessarily provide information about transient phenomena. Here we demonstrate the possibility that Bose condensation of nuclei is such a transient phenomena, which has thus far been unobserved. The aim of this Rapid Communication is twofold: first, to establish the possibility of Bose condensation of nuclei and the concomitant phase transition in nuclear physics; second, to suggest some specific experimental observations for its verification. The smallness of the finite volumes being considered could mask a clear signal of the phase transition. However, by exercising sufficient care, experimental signatures for the onset of Bose condensation may be observable.

Bose condensation of pions was predicted earlier [6] to occur in the expansion phases of nuclear collisions. There is, however, a subtle difference between Bose condensation of nuclei and that of pions. In the formation of nuclei, one requires several nucleons to be present in close physical and momentum proximity. Therefore, matter present must have some minimum density for condensation to occur. On the other hand, pion production is basically a one-nucleon phenomenon and therefore does not depend upon the presence of a minimum density of nuclear matter. Hence, pion condensation is observable in end-point measurements, such as total yields or cross sections, but condensation of nuclei is not. Its observation must be related to pre-ultimate stages of rarefaction (e.g., compression).

To focus on the possible existence of the phenomenon, a simple model is assumed, which ignores interactions between the particles. Such a model should be reasonable for the low densities and temperatures considered herein. More detailed and reliable predictions would certainly require a more sophisticated and realistic model; nevertheless, a simple model will suffice to demonstrate the possibility of observing this phenomenon.
We begin by considering an initial assembly of $N_{0}$ neutrons and $Z_{0}$ protons which have been compressed and are evolving in thermodynamic equilibrium at an excitation energy (temperature) $T$ and density $\rho$ to form light ions: ${ }^{12} \mathrm{C}$, ${ }^{11} \mathrm{~B},{ }^{10} \mathrm{~B},{ }^{9} \mathrm{Be},{ }^{7} \mathrm{Li},{ }^{6} \mathrm{Li},{ }^{4} \mathrm{He}(\alpha),{ }^{3} \mathrm{He}(h),{ }^{3} \mathrm{H}(t),{ }^{2} \mathrm{H}(d)$, together with neutrons $(n)$ and protons $(p)$. (Although the existence of a fully equilibrated system in heavy ion collisions is still debated, it is generally believed $[4,5,9-12]$ that equilibrium thermodynamics is a viable theory which gives reliable predictions.) In the model, charge and baryon number are conserved by requiring that

$$
\begin{aligned}
& \sum_{i} z_{i} N_{i}=Z_{0}, \\
& \sum_{i} n_{i} N_{i}=N_{0},
\end{aligned}
$$

where the $N_{i}$ refer to the number of particles of species $i$. In equilibrium, the chemical potential is

$$
\mu_{i}=z_{i} \mu_{p}+n_{i} \mu_{n}+\varepsilon_{i}
$$

where $z_{i}$ and $n_{i}$ are the number of protons and neutrons in the $i$ th species, and $\varepsilon_{i}$ is its binding energy. These particles have finite dimensions and initially move within a volume $V_{0}$. For convenience, we treat the particles as point particles (no physical dimensions) which move in a reduced volume given by

$$
V_{\mathrm{pt}}=V_{0}-\sum_{i} N_{i} V_{i}
$$

where $V_{i}$ is the eigenvolume of the $i$ th particle. The number (matter) density is

$$
\rho=\left(N_{0}+Z_{0}\right) / V_{0}
$$

The density of point particles is

$$
\rho_{\mathrm{pt}}=\left(N_{0}+Z_{0}\right) / V_{\mathrm{pt}},
$$


where $\rho_{\mathrm{pt}}>\rho$ since $V_{\mathrm{pt}}<V_{0}$. Our results will be presented in terms of $\rho_{\mathrm{pt}}$.

For a distribution of fermions, quantum statistics [13] yields

$$
N_{i}=2 g_{i} V_{\mathrm{pt}} \pi^{-1 / 2} \lambda_{i}^{-3} F_{\mathrm{FD}}\left(\mu_{i} / k_{B} T\right)
$$

where $g_{i}=2 S_{i}+1$ is the spin degeneracy, $\mu_{i}$ is the chemical potential, $\lambda_{i}$ is the thermal wavelength for the $i$ th particle of mass $m_{l}$,

$$
\lambda_{i}=2 \pi \hbar\left(2 \pi m_{i} k_{B} T\right)^{\cdots 1 / 2},
$$

where $k_{B}$ is Boltzmann's constant, and the species index is $i$ $\left(=p, n, h, t,{ }^{7} \mathrm{Li},{ }^{9} \mathrm{Be},{ }^{11} \mathrm{~B}\right)$ as appropriate. Values for the Fermi-Dirac integral functions

$$
F_{\mathrm{FD}}(\nu)=\int_{0}^{\infty} \frac{x^{1 / 2}}{1+e^{x-\nu}} d x
$$

are tabulated in the literature [14] and are increasing functions of $\nu$. For bosons $[13,15,16]$, we have

$$
N_{i}=\left[\exp \left(-\mu_{i} / k_{B} T\right)-1\right]^{-1}+g_{i} V_{\mathrm{pl}} \lambda_{i}^{-3} F_{\mathrm{BE}}\left(-\mu_{i} / k_{B} T\right)
$$

where the first term on the right-hand side of Eq. (9) gives the number of condensed bosons, the second term is the number of noncondensed bosons, and the species index is $i$ $\left(=d, \alpha,{ }^{6} \mathrm{Li},{ }^{10} \mathrm{~B},{ }^{12} \mathrm{C}\right)$. In Eq. (9), the Bose-Einstein integral functions [15] are given by

$$
F_{\mathrm{BE}}(\nu)=\int_{0}^{x} \frac{x^{1 / 2}}{e^{x+\nu}-1} d x
$$

The evolution of the system is studied by self-consistently solving Eqs. (6) and (9) while simultaneously requiring Eqs. (1) and (2) to be satisfied, until a convergent solution has been found for a specified temperature and density $\rho_{\mathrm{pt}}$. The density is then increased for that temperature until another convergent, self-consistent solution is found. Using this procedure, we find that there is a maximum density, for each temperature, beyond which no self-consistent solution exists. This occurs when the magnitude of the chemical potential for

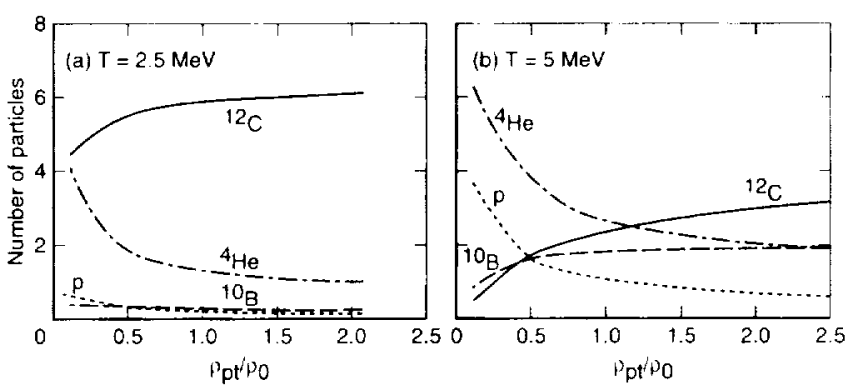

FIG. 1. Distribution of most abundant particles up to densities of $2.5 \rho_{0}$. Curves for temperatures of 2.5 and $5 \mathrm{MeV}$ are displayed. Other species have abundances which are smaller than the lowest curve shown here.

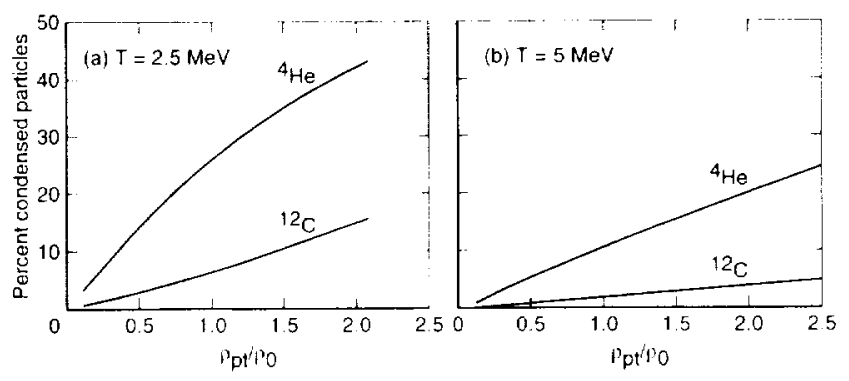

FIG. 2. Percentage of condensed bosons as a function of density ratio at temperatures of 2.5 and $5 \mathrm{MeV}$.

one of the bosonic species (usually $\alpha$ or ${ }^{12} \mathrm{C}$ ) becomes small leading to Bose condensation and a concomitant phase transition.

To illustrate the application of these methods, consider a model system, of $N_{0}=40$ neutrons and $Z_{0}=40$ protons, such as might exist in a ${ }^{4(} \mathrm{Ca}-{ }^{41} \mathrm{Ca}$ central collision. Figure 1 displays particle distributions for $T=2.5$ and $5 \mathrm{MeV}$ as a function of the density ratio $\rho_{\mathrm{pt}} / \rho_{0}$ where $\rho_{0}=0.17 \mathrm{fm}^{-3}$. Note that the ion distributions vary substantially with $T$ or $\rho$. From these results it is clear that bosons are more abundant than fermions. The most abundant ion species are ${ }^{12} \mathrm{C}$, ${ }^{10} \mathrm{~B}$, and ${ }^{4} \mathrm{He}$. Figure 2 displays the percentage of condensed particles for $T=2.5$ and $5 \mathrm{MeV}$. From these results we note that there is a finite probability of producing condensed alpha particles and condensed ${ }^{12} \mathrm{C}$ nuclei at densities occurring in heavy ion collisions $\left(\rho \leqslant 2 \rho_{0}\right)$, and even for densities below that of normal nuclear matter $\left(\rho<\rho_{0}\right)$. Also, the percentage of condensed bosons increases with increasing density. Figure 3 displays the chemical potentials for the most abundant bosons. All other boson species have values which are smaller (more negative) than those displayed in the figure.

From the results of these analyses with a simple, yet realistic physical model, it is apparent that Bose condensation of nuclei, such as ${ }^{4} \mathrm{He}$ or ${ }^{12} \mathrm{C}$, is a real possibility in central collisions of heavy ions.

The question is, how do we observe it? Although the most favorable conditions (for producing condensed bosons) of high densities and low temperatures (preferably less than 8 $\mathrm{MeV}$ ) may be difficult to achieve in laboratory experiments, the reasonably large percentage of condensed particles, indicated by our calculations, may provide a measurable signa-

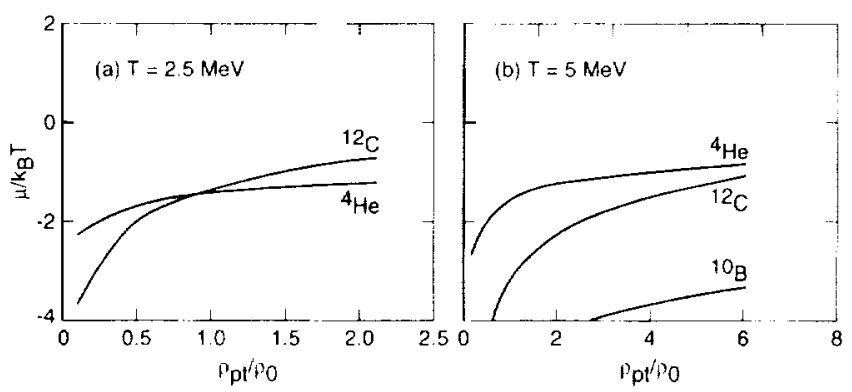

FIG. 3. Chemical potential as a function of density ratio for the most abundant bosons. Curves for temperatures of 2.5 and $5 \mathrm{MeV}$ are displayed. Other bosons have values smaller than those displayed here. 
ture in more realistic laboratory conditions. The most common observable for any distribution of particles is to measure total yields or cross sections. A measurement of the total yields, however, would not confirm or deny the existence of condensed bosons. From Fig. 1, we note that the total yietd of bosons (condensed and noncondensed) varies with temperature and density. From Fig. 2, the relative proportions of condensed to noncondensed bosons also varies. Consequently, experimental observation or detection must be based upon methods which distinguish between these two categories of bosons. One such method of detection might involve measuring the momentum distributions of a boson species (e.g., ${ }^{12} \mathrm{C}$ or ${ }^{4} \mathrm{He}$ ). In the center-of-mass system, the condensed bosons will be produced with near-zero momenta, whereas the noncondensed bosons will have much larger momentum values. Therefore, finding bosons with near-zero momenta in the center-of-mass system would be a clear signal that Bose condensation has occurred. In the laboratory frame, these condensed bosons would be detected at $0^{\circ}$ to the beam with a momentum equal to that of the collision system center of mass. There should be no significant difficulty in detecting them [17].

Finally, we emphasize that the findings and conclusions of the present work, although definitive in nature, deserve further study and refinement using more sophisticated models than the one used here. Nevertheless, the specific signature indicates that experimental verification of our proposal should be possible even though difficult because of the finite sizes of nuclear ensembles. In view of the importance of the phenomena to studies in nuclear physics and astrophysics, verification of its existence or nonexistence merits concerted efforts by experimental investigators.

One of us (R.K.T.) gratefully acknowledges the support of the National Aeronautics and Space Administration. Useful discussions with Jack Miller and Ken Frankel (Lawrence Berkeley Laboratory), Gary Westfall (Michigan State), George Fai (Kent State), and Bary Malik (Southern Illinois University) are gratefully acknowledged.
[1] L. P. Csernai, Phys. Rev. Lett. 54, 639 (1985).

[2] J. Cugnon, Phys. Lett. 135B, 374 (1984).

[3] W. Bauer, G. F. Bertsch, and H. Shultz, Phys, Rev. Lett. 69, $1888(1992)$

[4] L. P. Csernai et al., in Procedings of the IV Nordic Meeting on Intermediate and High Energy Nuclear Physics, Geilo, Norway (University of Lund, Lund, Sweden, 1981), p. 20; P. R. Subramanian et al., J. Phys. G 7, L241 (1981).

[5] L. P. Csernai and J. Kapusta, Phys. Rep. 131, 223 (1986), and references therein.

[6] J. Zimanyi, G. Fai, and B. Jakobson, Phys. Rev. Lett. 43, 1705 (1979).

[7] A. D. Panagiotou, M. W. Curtin, H. Toki, D. K. Scott, and P. J. Siemens, Phys. Rev. Lett. 52, 496 (1984); P. J. Siemens, Nature 305, 410 (1983).

[8] R. W. Minich et al., Phys. Lett. 118B, 458 (1982); A. S. Hirsch et al., Phys. Rev. C 29, 508 (1984).
[9] G. F. Bertsch and S. Das Gupta, Phys. Rep. 16, 180 (1988), and references therein.

[10] H. Stöcker and W. Greiner, Phys. Rep. 137, 227 (1986), and references therein.

[11] R. K. Tripathi, Phys. Rev. C 25, 1114 (1982).

[12] A. DeAngelis and A. Z. Mekjian, in Relativistic Heany Ion Physics, edited by L. P. Csernai and D. D. Strottman (World Scientific, 1991), Vol. 6. p. 36.3; D. H. Boal, Annu. Rev. Nucl. Part. Sci. 37, 1 (1987); W. G. Lynch. ibid. 37. 493 (1987), and references therein.

[13] J. D. Walecka, Fundamentals of Statistical Mechanics (Stanford University Press, Stanford, 1989).

[14] A. C. Beer, M. N. Chatse, and P. F. Choquard. Helv. Phys. Acta 28, 529 (1955).

[15] J. E. Robinson, Phys. Rev. 83, 678 (1951).

[16] F. London, Superfinids (Wiley, New York, 1954), Vol. Il.

[17] G. D. Westfall, private communication. 
